\title{
A Future without Inertia is Closer than you Think
}

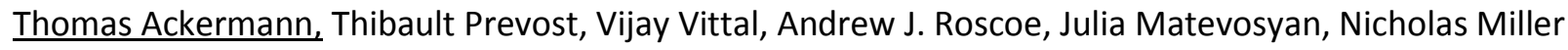

\section{Everybody knows Renewables are Coming}

Unless you have been hibernating in a remote cave for the past decade, you will have noticed the explosion of variable renewable generation. Wind power and solar photovoltaics have been the subject of dozens of articles, just within the pages of P\&E magazine. Charts showing relentless growth, such as the example from the US shown in Figure 1, with futures tending towards $100 \%$ renewable energy, are common. This figure is provided by NREL, and reflects a low cost, high renewable projection scenario. But closer inspection of this type of annual energy data alone doesn't support concerns about a zero inertia world. After all, look at the most extreme reaches of the figure in 2050. Only wind turbines and PV systems are converter based technologies, providing about $60 \%$ of the energy in the 2050 scenario. More than half of the energy comes from synchronous resources, and that's more than 30 years in the future. Isn't this article a bit premature?

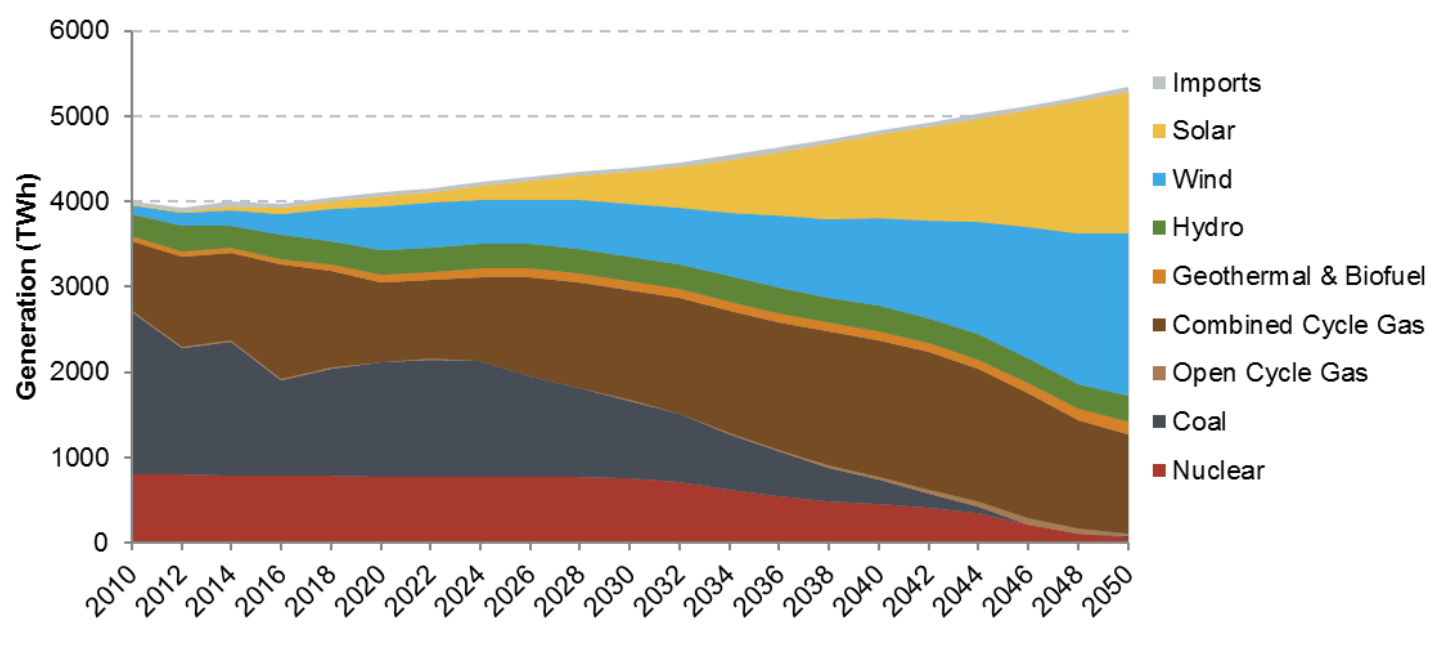

Figure 1: Renewable Energy Projections for the US Source: National Renewable Energy Laboratory. (2017).

\subsection{Remember: It's Power, not Energy}

The reality of wind and solar generation is well understood to be dominated by the availability of the resource; i.e. is it windy or sunny? Sometimes there will be a lot of wind and PV power available, when there is relatively little load. The concept of "penetration" is illustrated in Figure 2. This figure is looking at a near future scenario with enough wind generation to meet $30 \%$ of a study system's annual energy needs. The first dotted line is at $27 \%$, and represents the actual annual energy from wind as a 
fraction of the load energy consumed - for the year. The available wind energy was $30 \%$, but this system experienced curtailment of the wind during some operating hours because of dynamic security limitations. Moving to shorter time frames, the blue trace shows that some months are relatively windier than others, with the highest monthly penetration reaching about $32 \%$, and the lowest at about $22 \%$. Likewise, some weeks are windier than others (green trace). Some days are windier (orange), and some hours are windier (purple). This relentless trend is common to all systems with wind and solar. We can see here, that this particular $27 \%$ annual wind energy system exceeds $70 \%$ hourly penetration at times. This level of wind, or wind plus solar, is well within near term reach. For this system, increasing the annual wind energy to about $40 \%$, would result in many hours during which the desired operation of the system is at $100 \%$ (or greater, if export is possible).

Some power systems in the world already have situations with very high shares of converter based power supply, see box on current shares of non-synchronous sources.

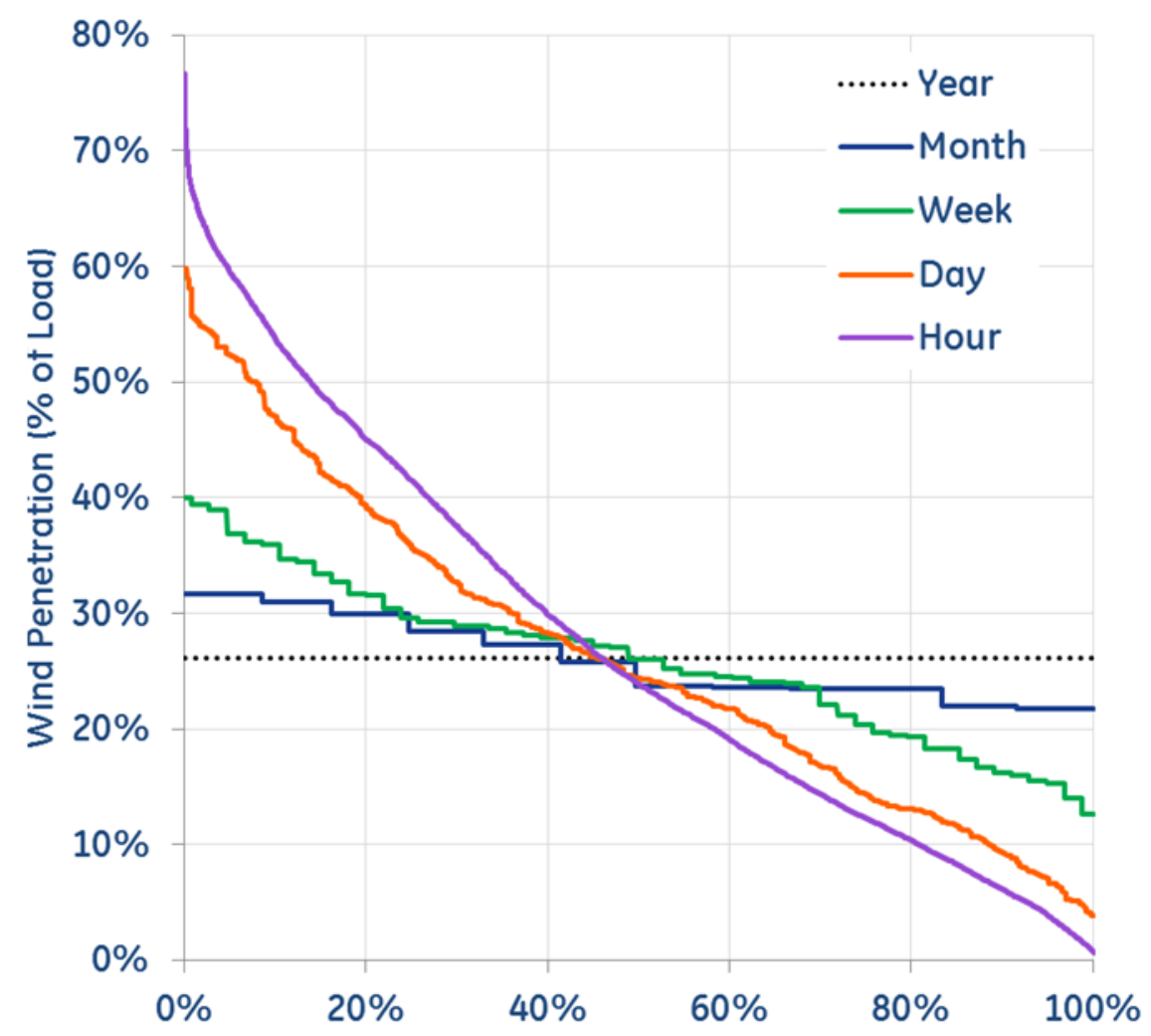

Figure 2 Wind Penetration Duration Curves for nominally 30\% Annual Energy System Source: NSPI Renewable Integration Study http://www.nspower.ca/site-nsp/media/nspower/CA\%20DR-

14\%20SUPPLEMENTAL\%20REIS\%20Final\%20Report\%20REDACTED.pdf 


\subsection{What about the Windy Sunny Neighborhood of the Grid?}

Another reality of wind and solar power generation, is that some places are better than others. In the case of wind power, location is critical to achieving high capacity factors (and therefore good return on capital investment). Similar considerations drive utility-scale PV installations. If we consider the representative system shown in Figure 3, a challenge presents itself. In this system, one corner has lots of good wind and solar generation, extremely limited economic synchronous generation, and limited transmission to the rest of the grid. A grid-wide look at the renewable penetration, might look like Figure 1, but the corner with lots of wind and solar, can easily and often reach or exceed $100 \%$ penetration of converter-based generation. What happens if that corner of the system disconnects from the rest of the grid? Today, the answer is "you can't run that island with 100\% converters".

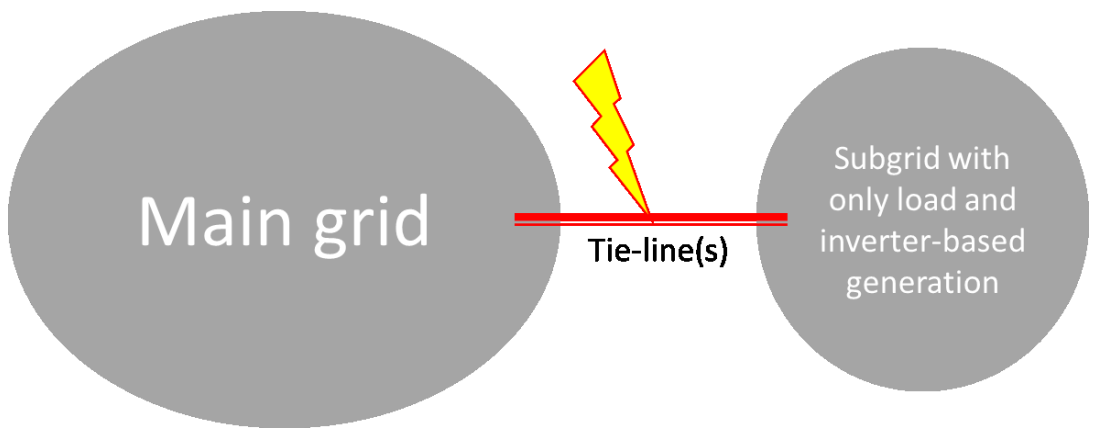

Figure 3: Fictitious grid with a high wind and solar extremity

The point of these simple examples is that the possibility to have actual operating conditions with zero synchronous inertia exist now, and will grow rapidly in the immediate future. As an industry we cannot wait until some distant future, when total renewable energy penetrations on an annual basis reach towards $100 \%$. There will be economic and reliability imperatives to be able to run with zero inertia upon much of the industry in the very near future.

In the rest of this article, we explore the challenge and options associated with this radical transformation of the grid. We start by examining present design and practice for the power electronics that enable wind and photovoltaics to deliver AC power to the grid.

\section{2. $100 \%$ Renewables isn't exactly the same as $100 \%$ Converters}

The rapidly emerging worlds of renewable energy and converter-based generation are tightly intertwined. A recent article in IEEE Power \& Energy, "Achieving a 100\% Renewable Grid", looked at a spectrum of issues. But while most wind generation and all photovoltaics rely on converters, many renewable resources deliver their power through synchronous machines. The most obvious is hydro power, but solar thermal, biomass, biogas, and geothermal also use synchronous generators. Further, we are not solely concerned with generation. It is understood that energy storage will likely play a role. At present the biggest energy storage resource worldwide is conventional pumped storage hydro (PSH), 
which uses synchronous machines. But rapidly growing energy storage technologies, including batteries, and variable speed PSH, use converters. Converters providing HVDC interconnection, static reactive compensation, converter serving loads, and even non-renewable generation that interfaces through converters (such as microturbines), all contribute to the growing trend of converter dominant systems.

\subsection{The Actual Behavior of Converters}

The main limitation that prevents converters from being the sole source of energy on a grid is the converter controls implemented today. Presently, grid-connected converters (even so-called "VoltageSourced" converters using forced-commutation devices) are controlled by software to operate as current sources that are grid-following. Physically, this means that converters provide a current that is shifted with the correct phase-angle with respect to the grid voltage to provide the desired active and reactive power. Practically, it means that these converters require "appropriate support" to provide the grid with a stiff voltage, which is presently done by synchronous machines. Eliminating all synchronous machines would mean that no frequency reference would be available to the grid and therefore grid-following converters would not be viable.

Hence it is essential for some of the converters to control the voltage. These converters are called grid forming converters. Their controls ensure that the voltage waveform of the grid is stable even at a very short time scale. This type of control enables the system to operate at a stable voltage even if loads connect/disconnect from the grid. Figure 4 illustrates the behavior of converter operating as a current source, when a load connects to it.

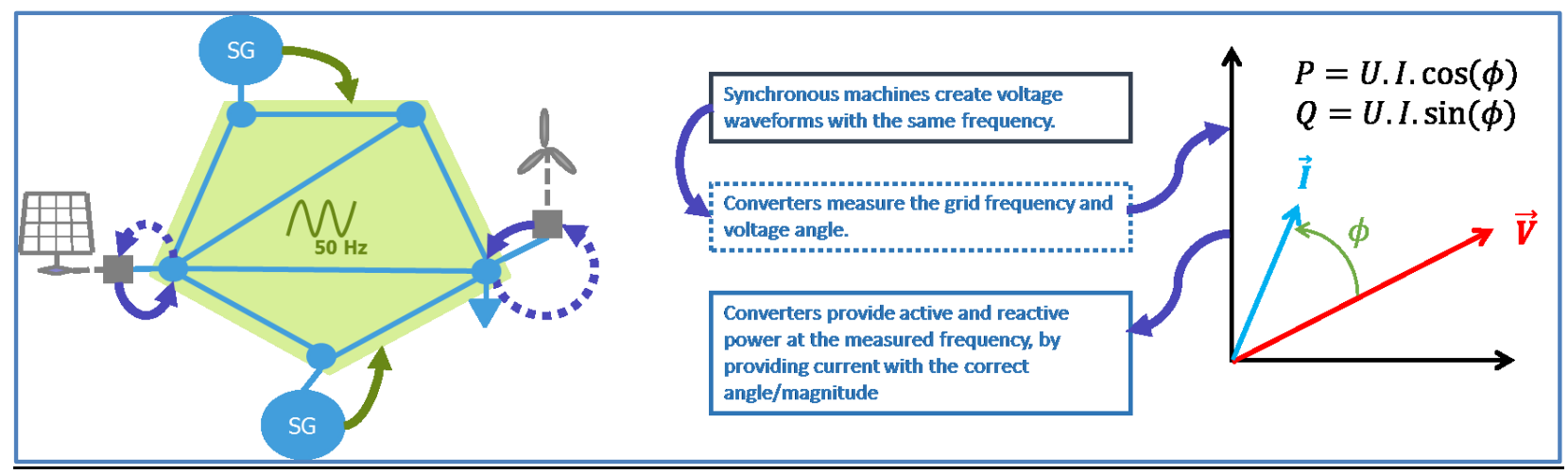

Figure 4: Actual grid operation with grid following converters(Source?)

\subsection{Changing of Electrical System Paradigm}

Going from a system driven solely by synchronous machines to a system with only power electronics introduces tremendous changes in the system dynamic characteristics. Synchronous machines are controllable in the steady state and dynamically within certain constraints which are set by physical 
limitations of the device. Additionally, in the case of synchronous machines the fast transient behavior is imposed by physical (both electrical and mechanical) characteristics of the machine. Therefore, regardless of the behavior of controls (e.g. excitation and governors), the system performance is largely predictable. With converters, the controls are much faster, and the behavior of such devices mainly depends on the characteristics of the control software. On one hand this feature of converters makes the system more controllable. On the other hand, the system is more vulnerable and dependent on the reliable design and functioning of the controls. Consequently, the specification for converters will need to be more precise and cover aspects that have never been examined for synchronous machines (shape of voltage during transients, fault current values and other similar characteristics).

\subsection{Frequency, from Analog to Digital}

System electrical frequency today is related to the rotor speed of synchronous machines. With converters however, the change is radical as frequency is a function of converter control. Frequency can vary rapidly, and even in a discrete fashion. Present equipment on the grid usually makes the assumption that frequency is continuous and slowly varying and will not function appropriately in a fast frequency varying environment.

\subsection{A Weak Grid?}

A weak grid is characterized by the short circuit current being "low". This definition can be seamlessly applied to synchronous machines because their behavior during a short circuit and in normal operation is guided by the same electrical characteristics of the machine. Different aspects need to be considered when considering weak grids with converters:

- Synchronous machines can provide extremely high current for a short period (1-100ms), where converters can normally provide little more than their nominal current. This would imply that the short circuit power will decrease. However, the issue is more complex:

- Often converters have a low loading factor. Therefore, the ratio between maximum current that can be provided by converters and the actual active power produced before a fault may not always be as low relative to the active power being produced.

- As an example, at initial loading of $20 \%$, which is a typical average load factor, the short circuit power that can be fed is around 5 times the active power delivered to the grid.

- With synchronous machines, the active power delivered to the grid is often close to their nominal power, under the assumption that synchronous machines are operated at maximum power, and the short circuit power delivered to the grid is 3-5 times the active power fed before the fault.

With regard to the stiffness of the network, the short circuit power is a good indicator, but for converters, regulation is the main driver of their behavior, and control in steady state and in fault mode can be radically different. As an example, the voltage regulation of a grid forming converter can create a very stiff grid voltage, and in the meantime controls can block the converter during fault mode, therefore providing no fault current. A new definition will probably be needed to address the grid stiffness with converters. 
The behavior of grid forming converters during a fault is one of the issues that will need to be carefully addressed, with one of the key aspects being the control of the voltage while maintaining current below the maximum converter capacity.

\section{Box: Current shares of non-synchronous sources:}

Many small power systems (up to $10 \mathrm{MW}$ peak demand) already achieve very high instantaneous nonsynchronous generation (NSG) penetration level of 60 to $80 \%$. Instantaneous NSG is thereby defined as generation from Power from Converters (plus HVDC imports if applicable) divided by Demand plus Exports for one hour.

The power system on the island of St. Eustatius, for instance, is using 1,9 MWp of PV and $1 \mathrm{MW}$ of batteries together with three diesel generators with a total capacity of $4 \mathrm{MW}$. PV and battery instantaneous penetration levels as high as $89 \%$ have been observed and the instantaneous penetration level will increase further as the PV system will be extended. Currently, even at high NSG penetration levels, a synchronous machines (diesel generator) operates at low output power, acting as synchronous compensators to provide solid voltage sources, while the converters provide the power. It is planned to upgrade the St. Eustatius so that it can be operated at certain times without any synchronous generator, i.e. a $100 \%$ instantaneous NSG penetration level could be achieved.

Also larger power systems already reach significant penetration levels. Tasmania is an island electrically connected to the mainland Australia by a monopolar HVDC interconnector (Basslink) rated at 500MW but offering dynamic rating of 630/480 MW (export/import). Tasmanian demand ranges from 900 to $1700 \mathrm{MW}$ and the generation sources include hydro $2250 \mathrm{MW}$, gas $380 \mathrm{MW}$ wind generation $300 \mathrm{MW}, \mathrm{PV}$ $96 \mathrm{MW}$ and $478 \mathrm{MW}$ HVDC import. The record NSG penetration level reached $78 \%$, considering Wind, PV and HVDC import.

In Ireland (5 GW peak demand, around $3 \mathrm{GW}$ wind power installed), the maximum NSG penetration level is currently limited by the system operator to $60 \%$, but it is expected that the limit will be increased stepby-step in the near future.

\section{Technology Options}

Grid forming converters, with appropriate controls both in the active power loop and in the reactive power loop, together with correctly designed droop characteristics, provide the ability to develop and operate inertia-less systems. The proof of concept can be demonstrated by means of large scale system simulation with effective models in positive sequence time domain simulations of large systems.

In a recent project completed by the Power Systems Engineering Research Center, the viability of a zero inertia power system has been systematically examined and analyzed. This viability analysis has been conducted on a large-scale system using the conventional industry practice of positive-sequence time 
domain simulation. A key initial step in this study was to develop an appropriate model for converter interfaced generation which would capture the essential characteristics of the actual device.

The primary focus of the study was on the possibility of control and operation of a zero inertia system; therefore, the reserve margin was not studied. It was found that the principles of power-frequency droop, coupled with the availability of fast response from the converter devices, can serve ably in both arresting frequency change and in the recovery of frequency.

The analysis conducted has shown that the control and operation of a zero inertia power system is viable. There is a primary assumption here that there is adequate active power margin to balance the system. For this to be the case with only converter-based renewable generation, some of the renewable sources of energy would need to be dispatchable or wide-spread use of energy storage would be needed.

The second assumption is that every converter interfaced generation source is capable of providing voltage support, which requires sizing of the converter to ensure that sufficient current is available to provide needed active and reactive current.

\section{Verification by simulation on a large-scale system}

Large-scale simulations of the Western Electricity Coordination Council (WECC), which includes all parts of the U.S west of the Rockies, the northern part of Baja California in Mexico and the Canadian provinces of British Columbia and Alberta, were performed. Amongst others, the following contingency case was examined using the inertia-less system model. The closing of a transmission line must be studied to ensure that it does not cause excessive transients of current and voltage. To observe this scenario, the power flow of the system was solved with a line between two major buses in Arizona outaged, resulting in a high angle difference between the buses. At $t=15 \mathrm{~s}$, the line was closed leading to a severe transient. With the maximum converter current set as $1.7 \mathrm{pu}$, Figure 5 and 6 show the converter currents for nearby generating units. From the figures it can be seen that though there is a large increase in the instantaneous current, the pre-disturbance current value is achieved within 1s. In addition, as expected, the unit located close to the line is affected to a much larger extent, but the system, is stable and secure. However, if the nearby generating units had a maximum current value of $1.4 \mathrm{pu}$, the unit located close to the line would trip while the other units pick up the slack. 


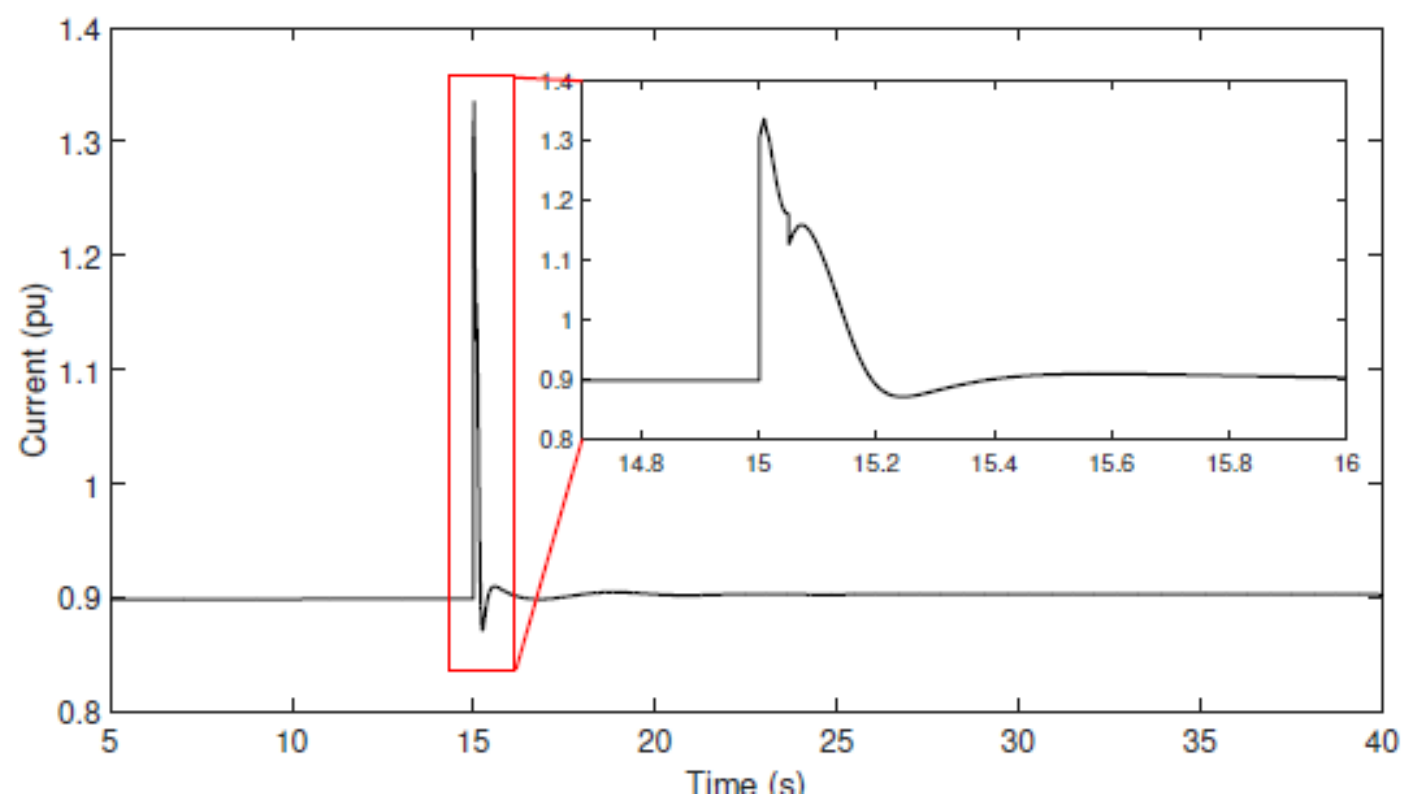

Figure 5: Converter current of one Plant A unit for the opening of a tie line between Arizona and Southern California following a line fault

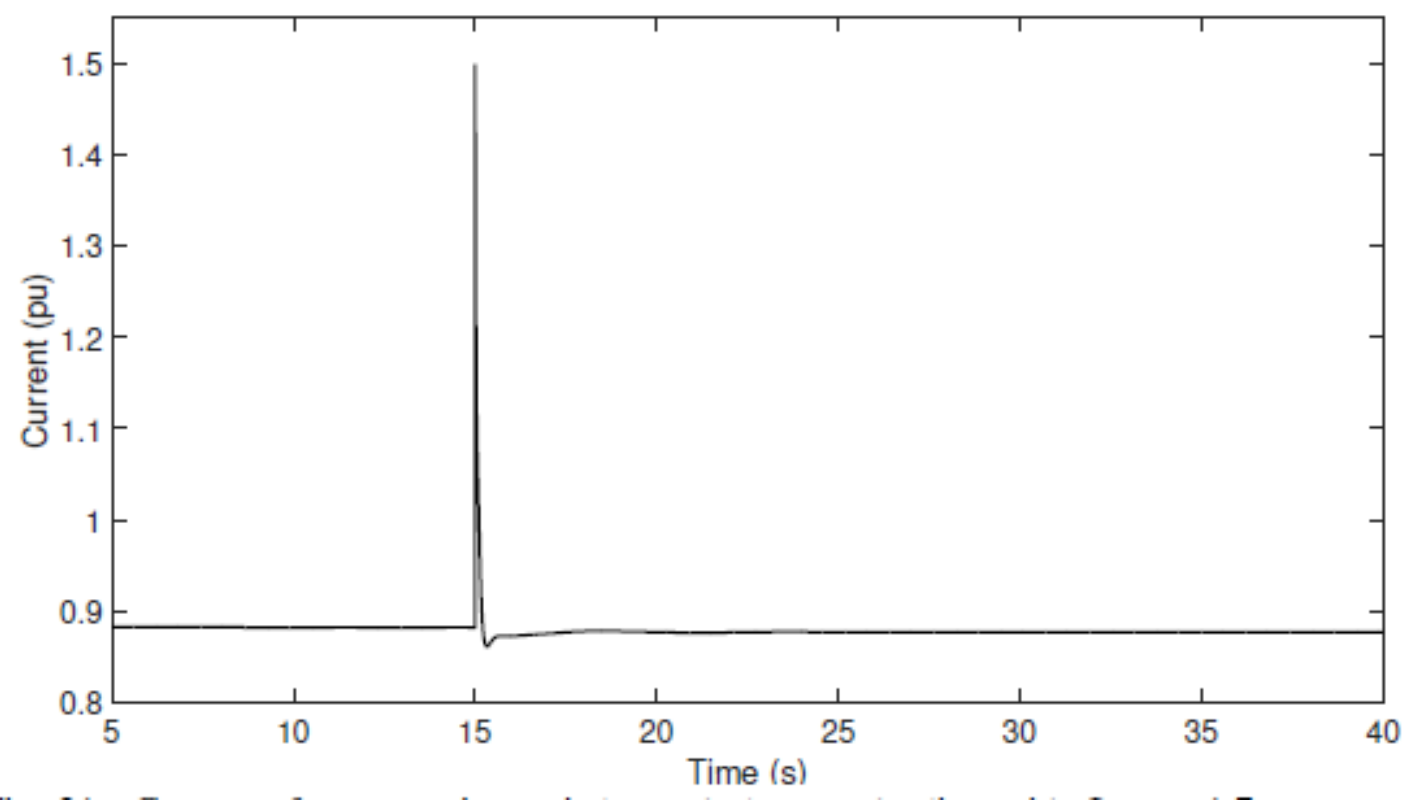

Fig. 6: Current of a generating unit located close to the line with Imax=1.7 
This large-scale simulation based exercise has demonstrated that the use of only grid-forming converters with well-designed control and adequate head room in the generation resources could work effectively even in large systems and tolerate large disturbances including significant loss of generation and closing of large transmission lines.

\section{Near-term Solution: Power Electronics must be able to provide Ancillary Services.}

In most countries, generation sources are required to provide some ancillary services. Usually the type of services depends on the size of the installation or the voltage level where it is connected. For transmission connected installations, it is now common to provide ancillary services like reactive power control and frequency response, but more services will be needed from converters when they are maintained in grid-following configuration.

- Volt/Volt Ampere Reactive (VAR) regulation: the adjustment of reactive power following a setpoint, or using a droop control that links the terminal voltage and the reactive power. This is today required for most of converters while they are producing active power. As active power of such installations is usually fluctuating, it will become more and more necessary that the reactive power capability does not depend on the active power output. Such a requirement is sometimes called STATCOM capability. It is a very important ability because it ensures a stable voltage at any time of the day (with or without sun/wind)

- Active power control:

- Frequency regulation: very similar to what is done presently for synchronous machines. There is a droop control between grid frequency and the active power delivered by the converter. The converter measures the electrical frequency and adjusts its active power accordingly. (This requires some reserve of active power to be able to deliver more power when frequency decreases.)

- Limited frequency control: similar to frequency control but with a large dead band that makes it act only when the frequency deviation exceeds a "normal" deviation (between 200 and $500 \mathrm{mHz}$ ). This usually has to be provided without keeping any reserve. (For units operated at maximum power, they will not provide any control in case of decreasing frequency.)

- Fast frequency control (so called synthetic or emulated inertial response). To limit frequency deviation, converters can provide short term frequency regulation. The energy that is provided to the grid can come from different sources: kinetic energy of a wind turbine, dedicated battery and other such reserves. The main difference between emulated inertia and actual inertia is that the emulation requires the frequency to be measured (see also box on Measuring Frequency); therefore its effect on the grid is delayed and for very fast frequency transients it may not be enough. 
- Alternatively, ancillary services for active power/frequency control can be defined based on specific system needs and procured thought market mechanisms. This would allow suitable technologies to provide the services in the most efficient way. For example, large industrial loads with underfrequency relays are capable of providing fast, effective and sustained response during under frequency events.

- Specific fault ride through behavior: formerly converters used to disconnect when the voltage was too low; they are now able to provide current even during faults. Moreover, the current is fully controllable, and it can be tuned to provide active only, reactive only or any configuration of both. This can be tuned to limit the voltage drop on the grid during a fault transient. Nowadays converters are even able to provide unbalanced current during faults for specific needs like protection.

Other mitigation measures, such as power production limit due to weak grid concerns (e.g. in ERCOT), non-synchronous generation penetration limit and/or inertia limit (e.g. in Ireland), use of synchronous condensers throughout the network, etc. can be applied in the interim to maintain system reliability. - 


\section{Long-term Solution: To push the limit of Converter Penetration up to $100 \%$ new Controls will be Necessary}

The first capability is for converters to be grid forming. One of the consequences of being grid forming is that the output power of the converter is now driven by the AC grid and not directly by the primary source. From a consumer's point of view, it is a basic requirement, to be sure that when a new load is connected, it will be fed by the grid. From a producer's point of view, this is more challenging. The converter is basically a link between an uncontrollable load and varying but predictable primary energy. Controls can change the operating point of the converters to adjust the output to the input, but during transients, some energy will be needed leading to the requirement for some type of storage or head room. (The amount of storage or head room will depend on the speed of the controls; small sized devices could be sufficient.)

\section{Protecting the converter against high current.}

Voltage controlled converters are prone to overcurrent during grid transients. In case of a grid transient, if the voltage angle shifts, it leads to a shift between active and reactive power but the total current remains constant and limited to the maximum value. When converters are operated in the voltage source mode, the active and reactive power that flows out is guided by the network voltage amplitude and phase before the action of the controls. The apparent power of the converter has very limited impact on it, and network modification due to topology changes can lead to high overcurrent as illustrated in the section on near-term solutions.

Constraining transients for a grid forming converter can be tie line opening and closing, or "large" load connection/disconnection close to "small" converters.

\section{Synchronization of converters and "Virtual Synchronous Machines"}

The synchronous torque inherently synchronizes rotating machines, but this is not obvious for converter based generation. The control must achieve synchronization of every unit, with changing conditions and most importantly without measuring frequency at least for some of them (as previously said, some converters need to form the grid). This synchronization can be achieved with two main varieties of gridforming converter control algorithm. The first variety emulates a synchronous machine, and is commonly referred to as a Virtual Synchronous Machine (VSM). The conventional grid-following converter current control loop architecture has a control bandwidth significantly in excess of $50 \mathrm{~Hz}$ and aims to source sinusoidal balanced currents in synchronism with the existing grid voltages, using a phase locked loop (PLL). It is possible to completely replace this control architecture with one that instead mimics a physical synchronous machine rotor. Only a generic representation is required, so that parasitic effects such as saliency and non-linearity are not crucial. The critical model parameters are rotor inertia, rotor electrical damping, and the transient impedance $X^{\prime}$. In practice, $X^{\prime}$ is equivalent (and can be defined) by the converter filter inductance which behaves almost exactly as a real machine $X^{\prime}$. The simulated rotor dynamics form a $2^{\text {nd }}$-order transfer function with a resonant frequency (normally) 
around $2 \mathrm{~Hz}$ for reasonable values of inertia $\mathrm{H}$ (in seconds), damping, and $\mathrm{X}^{\prime}$, just like a real machine. It can be shown that if you need to provide a response closely approaching true inertia from a converter, then you also have to accept that there will be a damped resonance. It may be possible to configure higher levels of damping than a real machine provides, even up to critical damping, since in the converter, damping is implemented mathematically and is not constrained by physical damper winding design or efficiency losses. However, more research is needed to fully understand the ramifications of high damping levels.

The VSM needs to have a suitable AVR (automatic voltage regulator) control loop applied, which provides voltage and reactive power control just as a real machine. A governor is also required which can take many forms, either following active-power setpoints, controlling frequency, providing droop response, or any combination. Optionally, "slow" prime mover responses can be simulated, i.e. steam turbine responses. Exactly what governor and prime mover models to use for a particular scenario requires more research, especially in the context of renewables, to examine the energy and power flows against time, and the requirement for short-term or long-term energy stores to ride through dynamic events.

The VSM behaves as a voltage source, since both its rotor dynamics ( $2 \mathrm{~Hz}$ bandwidth) and AVR dynamics have bandwidths $<50 \mathrm{~Hz}$, and the adjustments to the pulse width modulation (PWM) patterns are slow relative to the $50 \mathrm{~Hz}$ fundamental. It therefore does a very good job of "mopping up" unbalance, and it can provide power to heavily unbalanced loads. It is also quite good at "mopping up" harmonic voltages, although linear load is also (or more) effective at high harmonic orders. Because the VSM is a voltage source, during the closest faults, some intervention is needed to protect the solid-state devices from overcurrent. Viable methods have been demonstrated in the laboratory, able to sustain $>140$ ms full-depth balanced and unbalanced faults, but without compromising the normal "voltage source" capability. Additional interventions can be made during faults to (for example) set $\mathrm{H}=999$ during a fault, which helps to stop the virtual rotors accelerating while fault ride-through is active, and offers to make VSMs potentially more robust against faults, than real machines. Parameters such as damping, inertia and governor response can, if needed, be adjusted in real-time, remotely via software.

Because the VSM is a voltage source, with a frequency and voltage stabilized by its virtual inertia and AVR, it provides "synchronizing torque". It also provides inertia. But, crucially, it is not necessary to provide inertia to provide synchronizing torque. They are not the same thing. It is possible to provide "synchronizing torque" without providing inertia.

This can be achieved by a using a second variety of grid-forming (voltage source) converter control algorithm. This second variety implements frequency and voltage control loops which operate on a strict pair of droop slopes: active power to frequency, and reactive power to voltage. There is effectively an "instant" ( 10ms) governor/prime-mover response time. The converter simply measures its output power either over a short window such as 1 exact cycle (which provides good harmonic mitigation), or measures the instantaneous power output and applies low-pass filtering of the order of $<1$ cycle period, so that the control loop bandwidth is $<50 \mathrm{~Hz}$, normally perhaps in the $\sim 20 \mathrm{~Hz}$ region. Then, using a simple linear droop slope with configurable frequency and power setpoints, a "target" frequency is 
determined. This target frequency is within a few percent of nominal for normal setpoint and droop slope configurations. The converter simply advances its "virtual rotor" at this frequency and power synchronization is effectively achieved. A parallel loop operates on reactive power and voltage. Such a control scheme has acquired various names in the literature such as "Power Synchronization" or "VSMOH". This latter term refers to the fact that the response of this architecture can be shown to be mathematically identical to a VSM, but with $\mathrm{H}=0$, no direct rotor electrical damping, and an "instantly responding" (with1 cycle) governor/prime mover delivering "instant" droop response. While rotor electrical damping is proportional to rotor speed minus electrical stator frequency, droop response is proportional to electrical frequency minus setpoint electrical frequency. While they are different, the "instant" droop response actually provides a useful damping of grid frequency disturbance, and is extremely effective at limiting frequency nadir during events. It also provides what would be called "synchronizing torque", even though it has zero inertia, because it acts as a stiff, balanced voltage source, with a well-defined frequency close to nominal, behind a reactance (formed by its filter impedance). A network powered only by "VSMOH" converters is entirely viable. In this scenario, a discrete resistive load step results in a network frequency that transitions from one frequency to another frequency (defined by the droop slopes and setpoints), over a period of about 1 cycle.

So, while this VSMOH type of controller offers no inertia, it is entirely viable as a grid-forming solution as confirmed by the PSERC analysis, and can also be used in parallel with real machines, and VSM converters that do offer inertia. No special time-sensitive communications are required between the converters, so long as sensible configurations of set-points and conventional droop slopes are used to suit the network and the connected energy sources. Sufficient energy must also be available on the DC busses to drive the converters, and serve the loads.

In both the above modes "VSM" and "VSMOH", the converters are grid-forming, provide synchronizing torque, serve unbalanced loads and mitigate unbalanced voltages, and serve non-linear loads and mitigate harmonic voltages. The converter contribution to these services is, by default, inversely proportional to the magnitude of the effective filter impedance, in exactly the same way as a synchronous machine's contribution to these phenomena is inversely proportional to its transient reactance $X^{\prime}$. For a real machine, $X^{\prime}$ is inversely proportional to machine rating, and this is normally true for a converter filter impedance too. So, the device's contribution to power quality and synchronization torque is roughly proportional to its rating, since $X$ is normally in the region of $0.1-0.15 p u$ (per unit) for both machines and conventional converters. This presents some further challenges (and perhaps opportunities) for multi-level converters which may in the future have much lower inductive filter impedances than conventional converters.

\section{Closure and Prognostications}

Compared to traditional generation, converter-connected devices are limited in maximum current due to the use of solid-state power electronics. They are also limited in terms of active-power response due to application-specific limits of power and energy availability at the DC bus, particularly for renewable- 
energy sources. However, if these constraints are managed, it is possible to provide stable converterdominated or even converter-only power systems, able to serve dynamic real-world customer loads that have unbalanced and non-linear components. To do this, at least a certain proportion of the converters need to be "grid forming", acting as voltage sources (not current sources), mitigating power quality, and providing active power as the loads demand. To enable seamless dynamic power-sharing between converters, conventional frequency-power setpoints and droop slopes can be configured, with low update rates commensurate with conventional network practice. Depending on the grid-forming converter controller variety, it may offer Virtual Synchronous Machine functionality with inertial support, or it may offer "VSMOH" functionality that offers fast-acting droop response but no inertia. It is entirely feasible to operate a converter-only network with zero inertia and bounded frequency nadir excursions; this having been demonstrated in simulation and practical experiments. However, there is insufficient industry experience to establish the exact proportion of inverters that need to be gridforming to create viable systems. Conversion of existing commercial installations to become gridforming will be impractical or impossible for much of the installed base.

Since so many existing grid-connected devices and machines expect frequency to change relatively slowly, sudden removal of all inertia from an existing network overnight would be a bad idea, with (for example) unpredictable consequences for protection relays, and large power transients for directlyconnected pumps and AC machine loads. As noted in the introduction, the evolution of the system will likely be on a continuum towards lower levels of synchronous resources. Whatever strategies are adapted must be capable of working with some synchronous machines.

More likely, in the near to medium term we will require a managed balance of inertia-providing and non-inertia-providing devices, the choice for each device being made with consideration to the properties of the energy source connected. However in the long-term more holistic system planning approach is needed. Band-aid solutions applied in interim by system operators world-wide to maintain reliability may inadvertently limit further integration of converter-connected devices. Defining grid forming function as essential reliability service may attract new grid forming inverters to the market but may also just provide additional incentives for existing synchronous generation. Requiring grid forming converter control from new converter-connected devices could be another route, still it will take time until sufficient number of those is installed. More research is needed to bridge that implementation gap between available grid-forming converter technology and integration of this technology into a power system with its existing energy resources, protection systems, interconnection requirements, energy and AS market structures and other attributes.

\section{Recommended reading}

M. Yu, A. J. Roscoe, A. Dyśko, C. D. Booth, R. lerna, et al., "Instantaneous Penetration Level Limits of Non-Synchronous Devices in the British Power System," IET Renewable Power Generation, 2016. 
Pengwei Du; Weifeng Li; Xinda Ke; Ning Lu; Orlando A. Ciniglio; Mitchel Colburn; Phillip M. Anderson, "Probabilistic-Based Available Transfer Capability Assessment Considering Existing and Future Wind Generation Resources" , IEEE Transactions on Sustainable Energy, Year: 2015, Volume: 6, Issue: 4

Ramasubramanian, D., Z. Yu, R. Ayyanar, V. Vittal, and J. Undrill, "Converter Model for Representing Converter Interfaced Generation in Large Scale Grid Simulations," IEEE Transactions on Power Systems, Vol. 32, No. 1, pp. 765-773, January 2017.

Julia Matevosyan, Pengwei Du,: Inertia: Basic Concept and Impact on ERCOT Grid, 15th International Workshop on Large-Scale Integration of Wind Power into Power Systems, Vienna, November 2016.

Kroposki, et al., "Achieving a 100\% Renewable Grid," IEEE Power \& Energy Magazine, March/April 2017.

\section{Biographies}

Thomas Ackermann is chief executive officer of Energynautics GmbH in Darmstadt, Germany.

Thibault Prevost is a research Engineer at RTE, France.

Vijay Vittal is the Ira A. Fulton Chair Professor in the School of Electrical, Computer and Energy Engineering at Arizona State University.

Andrew J. Roscoe is a senior lecturer at the University of Strathclyde, Glasgow, UK.

Julia Matevosyan is Lead Planning Engineer at Resource Adequacy group of Electric Reliability Council of Texas, USA.

Nick Miller is Senior Technical Director at General Electric's Energy Consulting group in Schenectady, NY. 


\section{Measuring Frequency: Not as easy as you might think}

For more than a hundred years of utility practice, the speed of synchronous generators has been the proxy for grid frequency. Turbine-generator governors have used deviations in rotor speed as the measure of departure of the grid from 50 or $60 \mathrm{~Hz}$. Today, non-synchronous resources must measure frequency directly from the grid. Conceptually simple. But there are challenges. For example, when is a disturbance a change in frequency, and when is it something else? This figure is for a single phase of a $50 \mathrm{~Hz}$ voltage sine wave. In the middle of the signal, a 20 degree phase jump occurs, as might be associated with a transmission line switching event. The elongation of the half-wave means that the "frequency", in this case defined by time between zero crossing, has dropped dramatically to $45 \mathrm{~Hz}$ for the next half-cycle. Of course, a synchronous machine would filter this out to a negligible deviation. Suitable filtration of the signal here would do likewise. But be careful what you ask for: rapidly measuring frequency can have some strange outcomes.

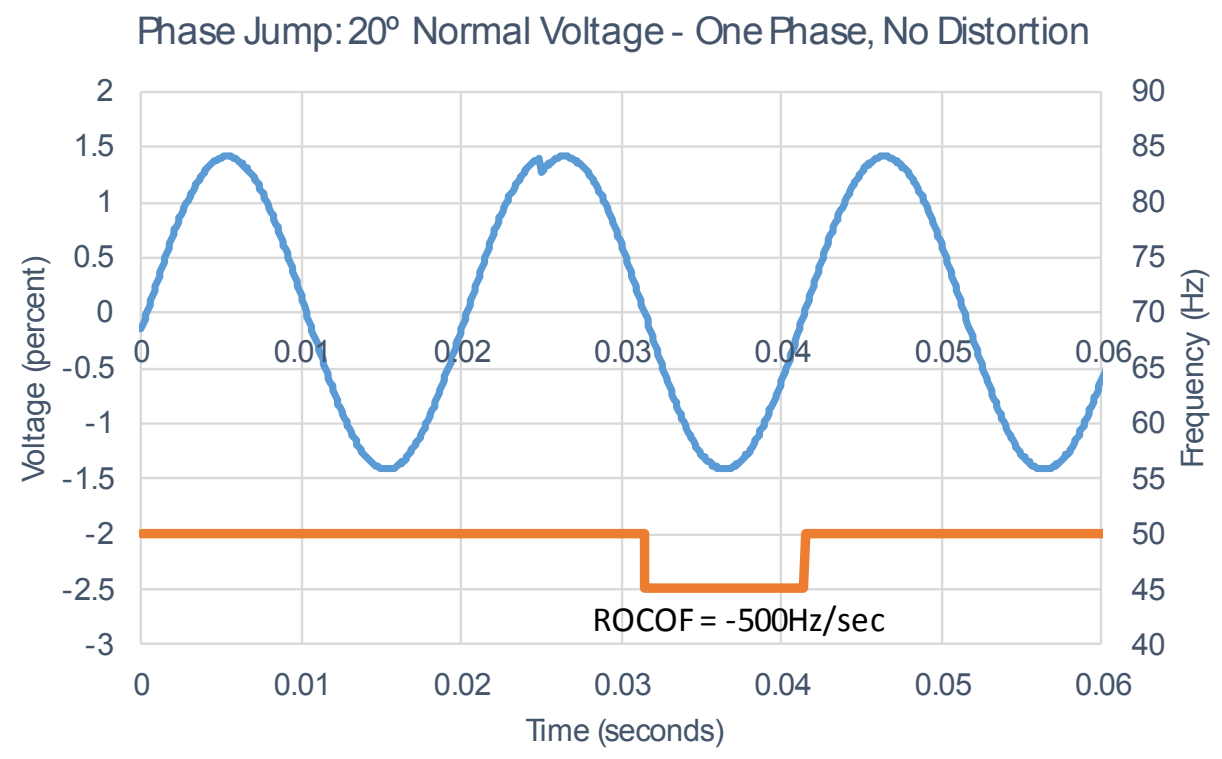




\section{Grid Forming Converters: a Twenty-year view from an Island.}

In the later years of the preceding century, the Native Alaskan community of Metlakatla faced a difficult technical and economic power system riddle: How to manage the extreme active power swings associated with the biggest load on their island grid? In order to displace an expensive, dirty diesel generator needed for regulation, a $1 \mathrm{MW}$-class utility-sale battery-converter systems was built. That system uses 1990's vintage self-commutating converters, with an early version of grid-forming, virtual synchronous machine controls. The system has been used to set frequency and voltage, black-start the grid, and manage a variety of difficult grid dynamics. After 20 years, the system has saved the community millions in diesel fuel costs, while maintaining a cleaner and more reliable grid.

Figure: Wall of batteries at Metlakatla Power \& Light installation, c 1996. (N.W. Miller, R.S. Zrebiec, R.W. Delmerico - GE, G. Hunt - GNB , Darrel Pierce Metlakatla P\&L; "Battery Energy Storage System for Metlakatla Power and Light," International Confe

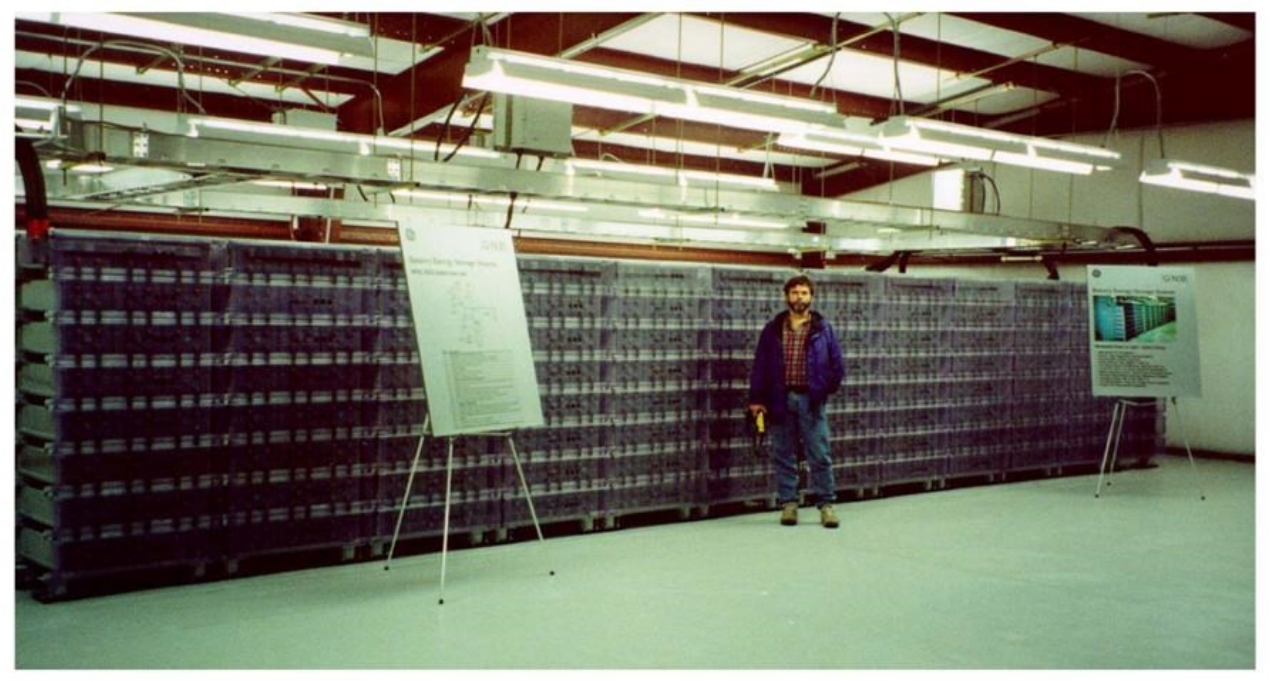

rence on Batteries for Energy Storage, San Juan, PR; 7/95) 
